\title{
Massive MIMO: A Measurement-Based Analysis of MR Power Distribution
}

\author{
Andrea P. Guevara, Sibren De Bast, Sofie Pollin \\ Department of Electrical Engineering, KU Leuven, Belgium \\ Email:\{aguevara,sdebast,spollin\}@esat.kuleuven.be
}

\begin{abstract}
In this work, an indoor ultra-dense massive MIMO experiment is analysed to quantify the interference power that affects undesired users or victims when MR precoding is applied. To compare scenarios resulting in different favourable propagation conditions between users, the antennas are deployed as a Uniform Rectangular Array (URA), a Uniform Linear Array (ULA) and a Distributed Uniform Linear Array $(D-U L A)$. We study scenarios where multiple users are served simultaneously. At the same time, the ultra-dense set of possible user locations is sub-sampled on a grid with a variable distance between users ranging from $50 \mathrm{~mm}$ to $600 \mathrm{~mm}$. This work shows, on the one hand, that a URA antenna configuration provides the highest power to victim users and the worst power distribution towards target users. On the other hand, due to improved favourable propagation conditions for many user location pairs, the D-ULA topology reduces the power to victim users. Moreover, it guarantees that the power received by the target user will always be at least $3 \mathrm{~dB}$ higher than any other victim user even if the users are closer as $100 \mathrm{~mm}$, for our analysis based on an indoor data-set and up to three target users.
\end{abstract}

Index Terms-Massive MIMO, Experiments, Measurements, Leakage Power, Maximum-Ratio Precoding.

\section{INTRODUCTION}

The principle behind massive MIMO systems is the superposition of waves generated by a huge amount of antennas that can create strong signals targeting small regions in space [1]. A broad power region can be advantageous in the case of wireless power transfer (WPT), here multiple WPT sensors within this beam could harvest a part of the power that is targeted to the main user [2]. However, in the case of wireless communications a narrower beam will concentrate the intended transmit power to the desired user without spatial dispersion, which leads to a system with a higher energy and spectral efficiency.

In a massive MIMO system, multiple precoding techniques can be applied to boost the desired signal towards the intended region while possibly suppressing undesired ones. In addition, favourable propagation conditions or wireless channel correlation between users also plays an important role in precoding. Different factors influence these favourable propagation conditions as the antenna array configuration at the base station, the user locations and the propagation environment [3].

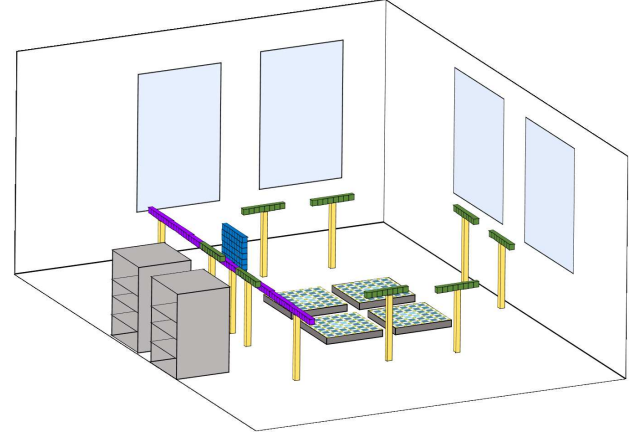

Fig. 1: 3D representation of the ultra-dense indoor Massive MIMO experiment. The grey boxes at the left represents the RF and processor components of the base station. Each antenna topology is presented per colour: URA - blue array, ULA - purple array, D-ULA - green arrays. The XY positioners, which hold a dipole antenna from each of the users equipment, are deployed on the floor and shown as the blue/green squares.

The state of the art provides a broad variation of precoding techniques, in this work we consider the low-complexity Maximum-Ratio (MR) precoder. The main idea behind MR is to coherently combine the desired signal, without any interference constraints [4]. Undesired signals can be suppressed due to orthogonality between channels as a consequence of favourable propagation conditions when the number of antennas goes to infinity [1]. In other words, the larger the number of antennas, the sharper or more focused the region where all signals add constructively. Focusing performance can be quantified by the amount of power that the target user receives in comparison to the power that is leaked to non-target users, named victims [5].

In this work, an ultra-dense indoor massive MIMO experiment is carried out by collecting the wireless channel for $\approx$ $252 \mathrm{k}$ locations distributed in an xy plane [6], to quantify the spatial MR beam directivity. As in real scenarios, the number of antennas in our testbed is limited to 64; therefore, we deploy three antenna array configurations to create scenarios with different degrees of favourable propagation between users. An analysis of the required distance between users is also considered as a parameter to measure the power received by victim users. 
In [2], the power transfer and leakage performance of a Massive MIMO system was measured experimentally for a range of locations and distances between target and victim users in pure line-of-sight conditions. Our work studies multiple antenna topologies and analyses the precoder performance based on the wireless channel for a large set of users. The data analysed is free for use and publicly available here.

This paper is organised as follows. Section III details the considered system model for the analysis, along with the definition of signal power for target and victim users. Section III] provides a wide description of the ultra-dense massive MIMO experiment, including the selection of users based on the distance between them. All the findings of this work are presented in Section IV] and the main conclusions are given at Section $\mathrm{V}$.

The following notations are used in this paper: $\mathbf{x} \in \mathbb{C}^{\mathrm{M}}$ represents an $M \times 1$ complex vector, $\mathbf{X} \in \mathbb{C}^{\mathrm{M} \times \mathrm{N}}$ is an $M \times N$ complex matrix. $\mathbf{x}^{\mathrm{T}}$ and $\mathbf{x}^{\mathrm{H}}$ state the transpose and conjugatetranspose of vector $\mathbf{x} .\|\mathbf{x}\|$ is the Euclidean norm of $\mathbf{x}$.

\section{Sytem Model}

In this work we consider a downlink (DL) time divison duplex (TDD) system with a total of $F$ subcarriers. This system is composed of a single-cell which has a single BS with $M$ centralised or distributed antennas, and $K$ single antenna users. The wireless channel per subcarrier $f$ between user $k$ and the base station is $\mathbf{h}_{k}(f) \in \mathbb{C}^{M \times 1}$, while the channel matrix is $\mathbf{H}(f)=\left[\mathbf{h}_{1}(f) \cdots \mathbf{h}_{K}(f)\right], \mathbf{H}(f) \in \mathbb{C}^{M \times K}$.

\section{A. Maximum Ratio Precoding}

After the channel estimation process, and before downlink transmission, the base station multiplies the downlink data to user $j$ by the precoder vector $\mathbf{w}_{j} \in \mathbb{C}^{M \times 1}$. In this case, Maximum-Ratio (MR) precoding to user $j$ is obtained as follows [7]:

$$
\mathbf{w}_{j}(f)=\frac{\mathbf{h}_{j}(f)}{\sqrt{\sum_{M}\left|\mathbf{h}_{j}(f)\right|^{2}}} .
$$

It is important to notice that in real scenarios, the mismatch between the estimated channel and the actual channel will influence the beamforming. During this study, we only have access to the estimated channel, therefore, the effects of these estimation errors on the signal power can not be assessed in this study.

\section{B. Target and Victim Signal Power}

The power from the precoded signal, which is intended to the reference user $j$, but is received to every other user $k$ in the system is obtained by [5]:

$$
\xi_{k \mid j}=\frac{1}{F} \sum_{f=1}^{F}\left\|\mathbf{w}_{j}(f)^{\mathrm{H}} \cdot \mathbf{h}_{k}(f)\right\|^{2} .
$$

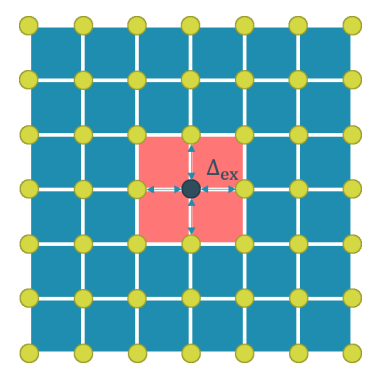

Fig. 2: User selection based on a grid location. All users must be discrete distance $\Delta_{\mathrm{ex}}$, in the $\mathrm{x}$ and $\mathrm{y}$-axis.

This allows us to quantify the amount of power that is leaked to victim users. Thus, when $j=k$, the "target signal power" is denoted as $\xi_{j}$. On the other hand, when $j \neq k$, $\xi_{k \mid j}$ is the "victim signal power". The best case scenario is when $\xi_{k \mid j}=0$, but this can only be achieved in a system when all the users have favourable propagation conditions towards the target user $j$ (i.e. mutual orthogonality among those vector channels, [3]). We sorted the $K-1$ victim users according to their leaking signal power, therefore the victim with the highest leaked power is called the "first victim user". In addition, we also considered the "leakage probability" $(\Upsilon)$ to quantify when the power seen by the first victim user is larger than the power seen by the target user:

$$
\Upsilon=\mathrm{P}\left[\xi_{j}<\max \left(\xi_{k \mid j}\right)\right] . \quad j \neq k
$$

\section{Multiuser Analysis}

In the case that MR precoding is applied to $L$ reference users, the precoding matrix $\mathbf{W} \in \mathbb{C}^{M \times L}$ per subcarrier $f$ is:

$$
\mathbf{W}(f)=\left[\begin{array}{ll}
\mathbf{w}_{1}(f) & \mathbf{w}_{2}(f) \cdots \mathbf{w}_{L}(f)
\end{array}\right] .
$$

The precoding vector of each of the $L$ reference users is combined to all the $K$ users in the system, to obtain $\mathbf{Z} \in$ $\mathbb{C}^{L \times K}$ :

$$
\mathbf{Z}(f)=(\mathbf{W}(f))^{\mathrm{H}} \cdot \mathbf{H}(f)
$$

Afterwards, all the signals given by the $L$ precoders are combined for all the $K$ users:

$$
\mathbf{z}_{k}(f)=\frac{1}{L} \sum_{l=1}^{L} \mathbf{Z}_{l, k}(f) .
$$

Here $\mathbf{Z}_{l, k}$ is this the combined matrix target to user $l$, and obtained by user $k$ ( $k$ could be target or victim user).

To finalise with the signal power vector, similar as (2):

$$
\xi_{k \mid L}=\frac{1}{F} \sum_{f=1}^{F}\|\mathbf{z}(f)\|^{2} .
$$

Here all the "target signal powers" are $k \in L$, while the remaining are the "combined victim signals power". 


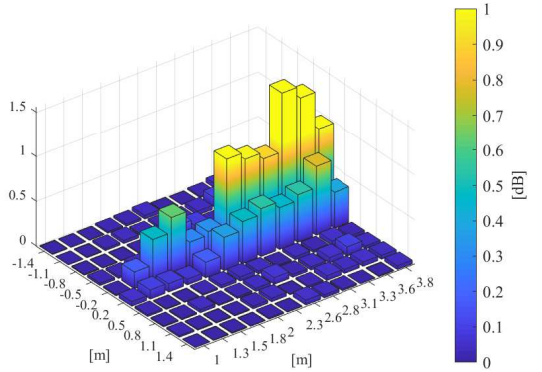

(a) URA topology.

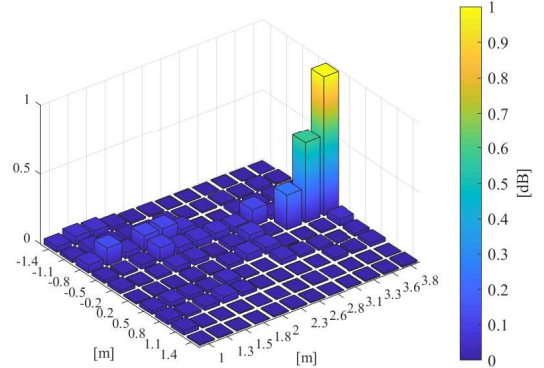

(b) ULA topology.

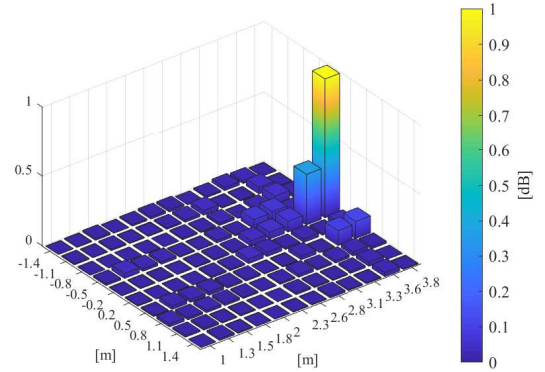

(c) D-ULA topology.

Fig. 3: Normalised spatial power distribution, representation in three dimensions for different antenna topologies when MR precoding is applied towards the user in $\mathrm{x}=-0.2 \mathrm{~m}$ and $\mathrm{y}=3.8 \mathrm{~m}$, while the centralised arrays are located at the origin.

\section{Measurement-Based Analysis}

In this section we describe the scenario, antenna array configurations, user distribution and data processing of the indoor massive MIMO experiment to measure the power of the target and victim users based on the wireless channel collection.

\section{A. Experimental Scenario.}

This experiment measures the wireless channel between multiple user positions and all the base station antennas, using the flexible and versatile KU Leuven MaMIMO testbed located at the department of electrical engineering (ESAT). This testbed is equipped with 64 antennas at the base station (BS) and is TDD-LTE based, controlled via the MIMO Application Framework of National Instruments [8] with a centre frequency of $2.61 \mathrm{GHz}$. In this experiment, we used 64 patch antennas at the base station $(M=64)$, that were deployed in three configurations:

- Uniform Rectangular Array (URA): All the antennas are deployed in a $8 \times 8$ array.

- Uniform Linear Array (ULA): 64 antennas placed next to each other to form a $64 \times 1$ array.

- Distributed- ULA (D-ULA): 8 sub-antenna arrays of $8 \times 1$ distributed over the room.

All the antennas were placed at the height of $1 \mathrm{~m}$, and $1 \mathrm{~m}$ distance from the area where the users were deployed, this scenario is depicted in Fig. 1 .

We use four user equipments with dipole antennas; those antennas were placed on top of 4 xy positioners, which were located on the floor. The positioners were automatically and synchronously moved in a grid area of $1250 \times 1250 \mathrm{~mm}$, inner squares in Fig. 1. The positioners move every $5 \mathrm{~mm}$ and wait for a short period, where the channel is collected at the base station from all user antennas deployed at the positioners. The total number of locations per configuration is 252004, and in each of those the wireless channel has a form of $\mathbf{h} \in \mathbb{C}^{64 \times 1}$, for each of the $F=100$ subcarriers.

\section{B. Users Density}

The position of the target and victim users influences the favourable propagation conditions of the scenario. In the experiment the distributions of the users is ultra-dense, therefore in this ideal scenario the user density quantified by the distance between users is also analysed. This distance is represented as $\Delta_{\mathrm{ex}}$, and it is displayed in Fig. 2. This scenario is created by sub-sampling the multiple locations from the wireless channel collected to satisfy $\Delta_{\mathrm{ex}}$. For the results presented in the following section, all the possible scenarios are considered for the range $50 \mathrm{~mm}<\Delta_{\mathrm{ex}}<600 \mathrm{~mm}$.

\section{RESUltS}

In this section, the results are presented in the following order: First, the signal power will be plotted for each user location with a fixed $\Delta_{\text {ex }}$ of 25 and $30 \mathrm{~mm}$ in the xy axis respectively, when one user is consider as target. Second, the signal power for the target and victim users is analysed for multiple $\Delta_{\text {ex }}$ values. Third, the probability of leakage $\Upsilon$ per antenna distribution is presented. Finally, the ratio between the signal power of the target and the first victim user is analysed.

\section{A. Spatial Signal Leakage}

In [1] the signal spatial distribution with MR precoding was simulated assuming a gaussian channel and with only a ULA antenna topology. On the other hand, in [9] the spatial MR precoding is also analysed only for D-ULA case, and in function of the number of active antennas.

To extend this study, the spatial signal power for multiple antenna distributions is analysed in Fig. 3 This graph in three dimensions presents the spatial location of the users in the $x y$ axis, while the $\mathrm{z}$-axis represents the normalised target signal power. In all the three antenna configurations, the same user is considered as the target one, which is placed at $\mathrm{x}=-0.2 \mathrm{~m}$ and $\mathrm{y}=3.8 \mathrm{~m}$. The distance between users here is $\Delta_{\mathrm{ex}}=$ $300 \mathrm{~mm}$ in the x-axis and $\Delta_{\mathrm{ex}}=250 \mathrm{~mm}$ in the y-axis. In 
the case of the centralised arrays (URA and ULA), the array centre is located at the origin.

In the URA scenario, Fig. 3a one can easily notice the fingerprint of the beam as the higher signal power of the users between the target user and the array of antennas. In some cases the signal power for undesired users is higher than the target one. Here, the first victim user is located in the beam and not immediately next to the target user and has a 50\% higher power in comparison to the target one.

The signal power for victim users drops massively when the ULA configuration is considered, Fig. $3 b$. Due to a rich multipath environment, most of the users between the antenna array and the target user have a power lower than $20 \%$ of the target users power. Nevertheless, the first victim user (which is located at $\mathrm{x}=-0.2 \mathrm{~m}$ and $\mathrm{y}=3.6 \mathrm{~m}$ ) has a signal power that is 0.58 times the target user power.

A reduction of the first victim signal power to 0.29 times the target user power is achieved when the antennas are deployed in distributed sub-arrays as the D-ULA in Fig. $3 \mathrm{c}$ here it is evident that the signal power to all the victim users drops. Without any doubt, it is clear in the set of the previous figures that the reduction of power to victim users is obtained as a consequence of a rich multipath scenario created by the distribution of the antennas.

\section{B. Signal Power Distribution and User Density.}

The results presented in the previous section consider a fixed distance between users $\left(\Delta_{\mathrm{ex}}\right)$, however, this separation also influences the power distribution. Fig. 4 shows the relation between $\Delta_{\mathrm{ex}}$ and the power distribution ( $\xi$ ). $\xi$ of the target user is presented in blue, while $\xi$ for the first victim user in red. We plot the interference mean power seen by those users in function of distance $\Delta_{\mathrm{ex}}$ and also show the power variation over all considered scenarios, as the width of the shaded area represents the first and third quartile. The solid lines are the mean power when a single user is considered as target, the dashed and dotted line shows the results when two and three users are served simultaneously. This analysis is carried out for the three antenna topologies considered in our experiment. It is worth mentioning that $\Delta_{\mathrm{ex}}$ is homogeneous for the $\mathrm{x}$ and $\mathrm{y}$-axis.

To obtain those values, first all the sub-sampled users are selected base on an equal distance $\Delta_{\mathrm{ex}}$, similar to Fig. 2 Then, in the case of a single target user, the target and the first victim signal power is computed, this process is carried out recursively for each user as the target one. In the case of two and three target users, the selection of those reference users and the estimation of the targets and first victims signal power $\left(\xi_{\mathrm{k} \mid \mathrm{L}}\right)$ are carried out over 10k iterations.

The first characteristic to be analysed is the target signal power, its mean (blue lines) and power distribution quantified as the blue area around the mean in Fig. 4, those values represents the power variations seen by all target users in

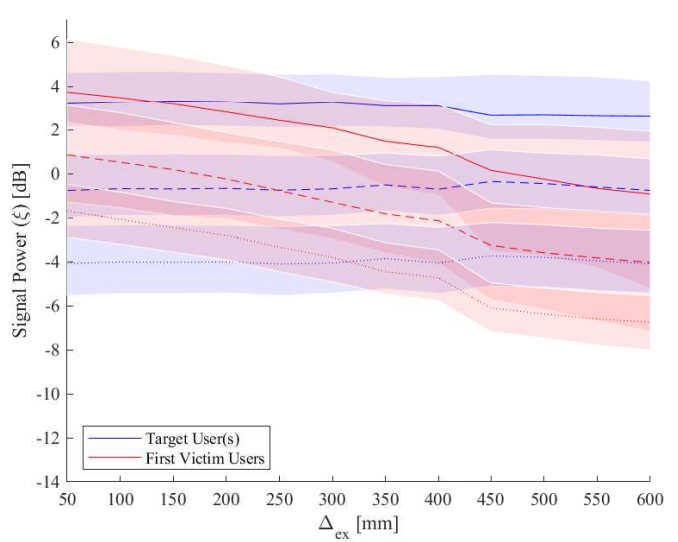

(a) Power distribution for two target users (blue) and first victim user (red), in a URA antenna topology.

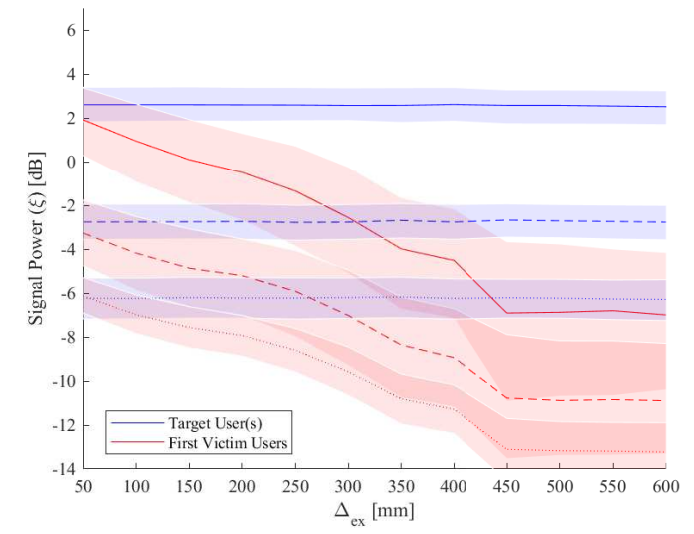

(b) Power distribution for a single and two target users (blue) and first victim user (red), in a ULA antenna topology.

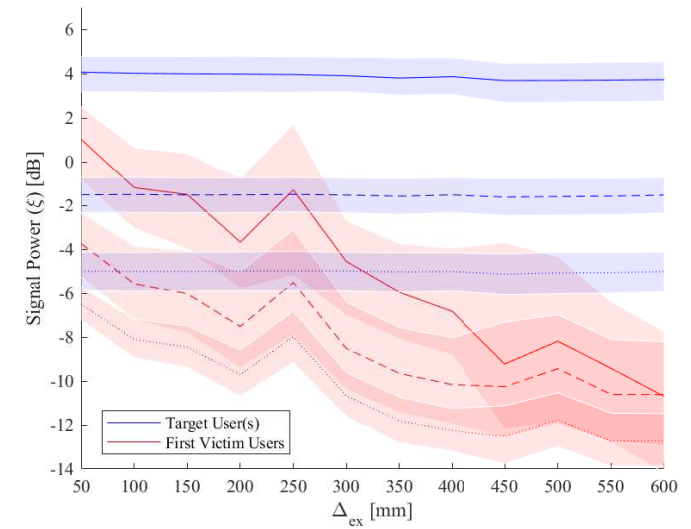

(c) Power distribution for a single and two target users (blue) and first victim user (red), in a D-ULA antenna topology.

Fig. 4: Mean and power distribution between the first and third quartile, when MR is applied to one (solid line), two (dashed line) and three (dotted line) users simultaneously, known as target users. The power distribution also includes the power distribution of the first victim user for a distance between users $\Delta_{\mathrm{ex}}$ variation between 50 and $600 \mathrm{~mm}$. 


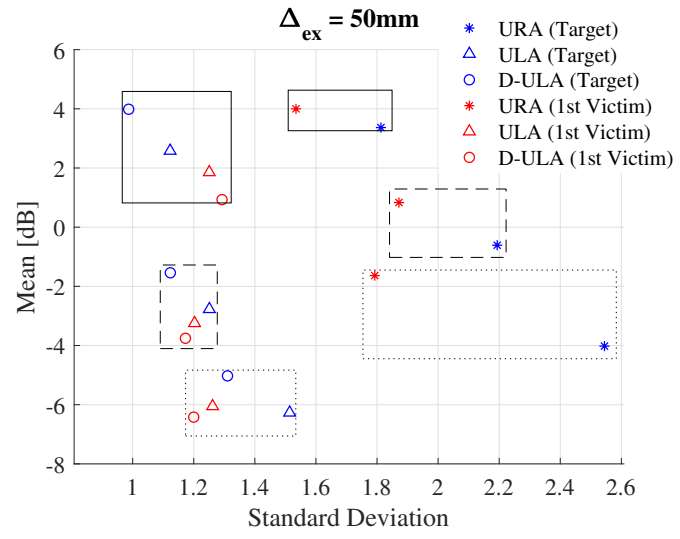

Fig. 5: Signal power standard deviation and mean power distribution for targets and first victim users. The values are grouped and limited by squares in solid lines when one user is consider as target user, in squares of dashed lines for two target users and squares in dotted lines for three target users. Those values are compared between multiple antenna topologies.

the room, depending on channel conditions. The mean target signal power remain constant to the variation of $\Delta_{\mathrm{ex}}$ but influenced by the antenna topologies and the number of target users. In our analysis, when two and three users are served simultaneously, the total transmitted power is distributed between them. Therefore as it is expected, there is a reduction in the mean reference signal power when the number of target users increases. See the solid, dashed and dotted blue lines.

In the case of the power distribution (blue area) there is a difference between antenna configurations, the URA case has $\approx 5 \mathrm{~dB}$ power variation which is the largest value in comparison with ULA and D-ULA, regardless the number of target users. This high power distribution is expected due to the significant distance variation between the users and the centralised array.

The second visible characteristic in Fig. 44 is the first victim signal power distribution presented as the red area and its mean as red line. Those values are decreasing with $\Delta_{\mathrm{ex}}$ and are antenna distribution dependent. On the one hand, only in the URA case, the first victim power mean (red line) is higher than the target one (blue line) when $\Delta_{\text {exc }}<150 \mathrm{~mm}$, $\Delta_{\text {exc }}<250 \mathrm{~mm}$ and $\Delta_{\text {exc }}<300 \mathrm{~mm}$ for one, two and three reference users, respectively. On the other hand, due to the favourable propagation conditions, ULA and D-ULA present a lower signal power to the first victim user. D-ULA is the configuration with the smallest power overlap between the reference and target user, in both cases when one, two and three users are served simultaneously. In other words D-ULA produces a sharper beam towards the target users.

\section{Signal Power Standard Deviation}

In Fig. 5 the mean values for the power distribution presented in Fig. 4 are compared to its standard deviation

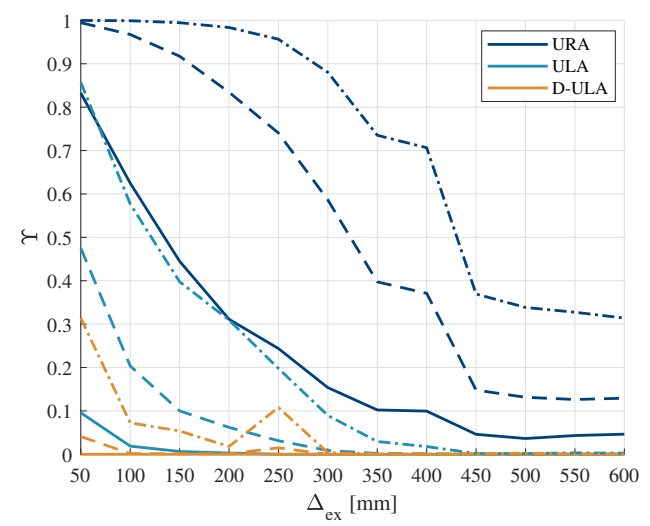

Fig. 6: Probability of leakage signal power to the first victim user being higher that the desired signal power with a variation of the distance between users $\left(\Delta_{\mathrm{ex}}\right)$, for three antenna topologies: URA, ULA and D-ULA. Solid lines presents single target user while dashed and dotted lines are two-users and three-users as target, respectively.

for a fixed user density $\Delta_{\mathrm{ex}}=50 \mathrm{~mm}$. The solid, dashed and dotted line enclosed the values when one, two and three users are served simultaneously (target users).

In this case the power standard deviation represents the signal power distribution, the lower the standard deviation, the more equal the power distribution. For the power distribution of the target users presented as the points in blue in Fig. 4, one can see that the standard deviation tends to increase if the number of target users also increases. However, the D-ULA configuration always has the lowest power standard deviation for target users in comparison with the other antenna configurations. However, the power standard deviation of the first victim users (red points) are only dependent on the antenna configuration but without any relation to the number of target users.

\section{Leakage Power Probability}

Fig. 6 shows the probability of the fist victim user having a higher received power than the target one $(\Upsilon)$, in function of the distance between users expressed as $\Delta_{\mathrm{ex}}$, and compared between the different antenna configuration. The solid lines assumes that MR is applied to a single user, while dashed and dotted lines when two and three users are served at the same time, respectively.

For the centralised topologies, $\Upsilon$ decreases exponentially in function of $\Delta_{\mathrm{ex}}$. But for a fixed distance between users, it is possible to reduce $\Upsilon$ with the antenna deployment. In the case that only one user is served, the reduction is from $82 \%$ in the URA case to $10 \%$ for ULA, considering the smallest $\Delta_{\text {ex }}$. However, when the number of target users increases, the probability gap between the same antenna distribution diminishes. 


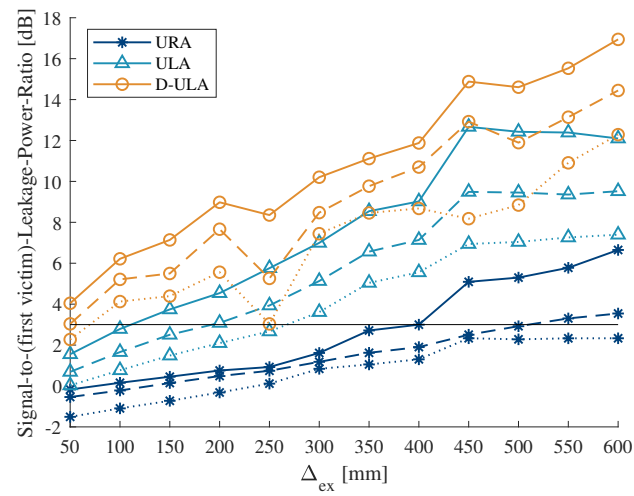

Fig. 7: Signal to (first victim) Leakage Ratio, over different distance between users. Solid lines consider a single target user, dashed two target users while dotted lines three target users.

Interestingly, for all $\Delta_{\mathrm{ex}}$ intervals considered in this experiment when only one user is served, the D-ULA configuration can guarantee that the target user will always get the higher transmitted power as $\Upsilon=0$. When two or three users are served, the D-ULA topology guarantees that all the target users will get a higher power than the first victim users when $\Delta_{\mathrm{ex}}>100 \mathrm{~mm}$ and $\Delta_{\mathrm{ex}}>300 \mathrm{~mm}$. The ULA does this when $\Delta_{\mathrm{ex}}>200 \mathrm{~mm}, \Delta_{\mathrm{ex}}>350 \mathrm{~mm}$ and $\Delta_{\mathrm{ex}}>450 \mathrm{~mm}$, for one, two and three target users respectively. The URA configuration can not guarantee the highest power towards the target user even if the distance between users is $600 \mathrm{~mm}$.

\section{E. Signal-to-(first victim)-Leakage-Ratio}

In Fig. 7 the ratio between the mean signal power of the target users and the signal power of the first victim user is presented. It is important to notice that unlike the full signalto-leakage-ratio (SLR) provided by the SoA we consider only the first victim user power for simplicity. To have the real SLR the power received by all undesired signals must be added.

When users are close to each other the power difference between desired and and undesired users decreases. To ensure a higher power difference, only a single user can be served (solid lines). In Fig. 7 the minimum distance is estimated for each antenna topology where there is $3 \mathrm{~dB}$ difference between the desired and leakage power. Thus, for a single target user, the distance between users must be at least $100 \mathrm{~mm}$ and $400 \mathrm{~mm}$ for ULA and URA, respectively, to obtain twice the desired signal power in comparison with the power obtained by first victim user. When two users are served simultaneously, the distance between users must be at least $200 \mathrm{~mm}$ and $500 \mathrm{~mm}$ to ULA and URA.

Notice that in the URA case, when three users are served simultaneously, the power difference between the mean power of the target users and the power of first victim user will not be higher than $3 \mathrm{~dB}$ even when distance between the users is as large as $600 \mathrm{~mm}$.

\section{Conclusions}

This paper studies the power distribution of an indoor Massive MIMO system with MR precoding. While a maximal power focusing performance is achieved for a single user case, we analyse also to what extent the power is spread evenly when increasing the number of users. First, it is shown that the D-ULA reduces the leaked power to victim users, while providing a better power distribution towards target users. Second, when two or more users are served simultaneously, there is not only a reduction of the power to the intended users but also a reduction of the power that is leaked to victim users as in general the power is more spread out in the environment. Third, for a single reference user, the D-ULA guarantees that the power of victim users will not be higher than the target ones. Even when the users are as close as $50 \mathrm{~mm}$, the power difference between the target and the victim user will be higher than $3 \mathrm{~dB}$. In overall, if the objective is to reduce the power leakage to other users and have a higher energy efficiency, we strongly suggest a DULA antenna topology for ultra-dense scenarios.

\section{ACKNOWLEDGEMENT}

This work was funded by the European Union's Horizon 2020 under grant agreement no. 732174 (ORCA project) and by the Research Foundation Flanders (FWO) SB PhD fellowship, grant no. 1SA1619N.

\section{REFERENCES}

[1] E. G. Larsson, O. Edfors, F. Tufvesson, and T. L. Marzetta, "Massive mimo for next generation wireless systems," IEEE Communications Magazine, vol. 52, no. 2, pp. 186-195, February 2014.

[2] S. Claessens, C. M. Chen, D. Schreurs, and S. Pollin, "Massive MIMO for SWIPT: A Measurement-Based Study of Precoding," IEEE Workshop on Signal Processing Advances in Wireless Communications, SPAWC, vol. 2018-June, pp. 1-5, 2018.

[3] H. Q. Ngo, E. G. Larsson, and T. L. Marzetta, "Aspects of favorable propagation in massive mimo," 2014, p. 76-80.

[4] T. L. Marzetta, E. G. Larsson, H. Yang, and H. Q. Ngo, Fundamentals of Massive MIMO. Cambridge University Press, 2016.

[5] A. Tarighat, M. Sadek, and A. H. Sayed, "A multi user beamforming scheme for downlink mimo channels based on maximizing signalto-leakage ratios," in Proceedings. (ICASSP '05). IEEE International Conference on Acoustics, Speech, and Signal Processing, 2005., vol. 3, 2005, pp. iii/1129-iii/1132 Vol. 3.

[6] S. D. Bast, A. P. Guevara, and S. Pollin, "Csi-based positioning in massive mimo systems using convolutional neural networks," in 2020 IEEE 91st Vehicular Technology Conference (VTC2020-Spring), 2020, pp. $1-5$.

[7] G. Ghiaasi, J. Abraham, E. Eide, and T. Ekman, "Effective Channel Hardening in an Indoor Multiband Scenario," International Journal of Wireless Information Networks, vol. 26, no. 4, pp. 259-271, 2019. [Online]. Available: https://doi.org/10.1007/s10776-019-00438-7

[8] E. Luther, "5g massive mimo testbed: From theory to reality," white paper, 2014

[9] A. P. Guevara, S. De Bast, and S. Pollin, "Weave and Conquer : A Measurement-based Analysis of Dense Antenna Deployments," pp. 1-6, 2020. [Online]. Available: http://arxiv.org/abs/2003.11467v1 